\title{
Aqueous microwave-assisted cross-coupling reactions applied to unprotected nucleosides
}

\author{
Gwénaëlle Hervé ${ }^{1}$ and Christophe Len ${ }^{1,2 *}$ \\ Sorbonne Universités, Université de Technologie de Compiègne, Ecole Supérieure de Chimie Organique et Minérale, EA 4297 Transformations Intégrées de la \\ Matière Renouvelable, Centre de Recherche Royallieu, Compiégne, France \\ ${ }^{2}$ Department of Chemistry, University of Hull, Hull, England
}

Edited by:

Stefano D'Errico, Università degli

Studi di Napoli 'Federico II', Italy

Reviewed by:

Rafael Luque, Universidad de

Cordoba, Spain

Daniele D'Alonzo, University of

Napoli, Italy

*Correspondence:

Christophe Len, Sorbonne

Universités, Université de

Technologie de Compiègne, Ecole

Supérieure de Chimie Organique et

Minérale, EA 4297 Transformations

Intégrées de la Matière

Renouvelable, Centre de Recherche

Royallieu, Compiégne, France

e-mail: christophe.len@utc.fr
Metal catalyzed cross-coupling reactions have been the preferred tools to access to modified nucleosides (on the C5-position of pyrimidines and on the C7- or C8-positions of purines). Our objective is to focus this mini-review on the Suzuki-Miyaura and on the Heck cross-couplings of nucleosides using microwave irradiations which is an alternative technology compatible with green chemistry and sustainable development

Keywords: nucleoside, nucleotide, Suzuki-Miyaura, Heck, water, green chemistry, microwave

\section{INTRODUCTION}

Nucleoside, nucleotide and oligonucleotide analogs exhibit, for some of them, antiviral and/or antitumoral activities and can be used as synthetic probes for biomedical applications. Numerous modifications have been made on C5- and C6-positions of pyrimidines as well as on C7-position of deazapurines and C8-position of purines. Very recently, literature even refers to modifications directly on oligonucleotides. In order to furnish greener solutions for chemical and pharmaceutical companies, the synthesis of nucleoside analogs via cross-coupling reactions has widely been studied. Cross-coupling reactions have mainly been developed in the presence of palladium-based catalysts via the Suzuki-Miyaura, the Heck and the Sonogashira reactions (Agrofoglio et al., 2003). Among the 12 principles of green chemistry, the use of benign solvents (water) and auxiliaries, the design of energy efficiency (microwave) and the use of catalysis were the most reported. In the particular domain of nucleoside chemistry, only the SuzukiMiyaura and Heck cross-coupling reactions were described in sole water under microwave irradiations.

\section{MICROWAVES ASSISTED SUZUKI-MIYAURA CROSS-COUPLINGS IN PURE WATER}

Fresneau et al. (2012) reported a Suzuki-Miyaura reaction starting from $2^{\prime}$-deoxyuridine in a completely aqueous medium using an inexpensive catalyst/ligand system which is also easily available. Model reaction conditions involved unprotected 5-iodo-2'-deoxyuridine and 4-methoxyphenylboronic acid. The best conditions consisted in the coupling of the unprotected chosen nucleoside in the presence of $\mathrm{Na}_{2} \mathrm{CO}_{3}$ (1.5 eq), $\mathrm{Pd}(\mathrm{OAc})_{2}$
$(3 \mathrm{~mol} \%)$ and $\mathrm{PPh}_{3}(5.4 \mathrm{~mol} \%)$ in neat water at $80^{\circ} \mathrm{C}$ for few hours. In the course of their study, Fresneau et al. (2012) decided to consider, under their above described conditions, the influence of microwave irradiation at $120^{\circ} \mathrm{C}$. This change in heating mode permitted a significant reduction in the reaction time ( $10 \mathrm{~min}$ vs. $4 \mathrm{~h}$ ) while keeping the same efficiency. Application of this new method to different arylboronic acids afforded 13 cross-coupling products in good yields with substrates that contained either activating or deactivating groups in the para and meta positions of the aromatic ring. The same year, Len's group reported an efficient ligand-free Suzuki-Miyaura cross-coupling reaction in pure water starting from free 5-iodo-2'-deoxyuridine (GallagherDuval et al., 2013). The catalyst system was composed of very low amounts of $\mathrm{Na}_{2} \mathrm{PdCl}_{4}(0.05-0.1 \mathrm{~mol} \%)$ and $\mathrm{KOH}$ (2 eq) (Scheme 1).

Various substrates with electron donating groups (EDG) and electron withdrawing groups (EWG) in the para or ortho positions (including sterically demanding boronic acids and heteroboronic acids) were systematically examined under both classical heating and microwave irradiations and 14 crosscoupling nucleoside derivatives were isolated (Gallagher-Duval et al., 2013). The coupling reactions were always more effective when the microwave irradiations were used. Three heteroboronic acids were also tested but it was found that only thiophen-2-boronic acid was reactive enough to give, in modest yields, the desired cross-coupling product using either conventional heating or microwave irradiation. Furan-2yl boronic acid was sufficiently reactive only using the alternative technology. 
<smiles>O=c1[nH]c(=O)n(C2C[C@H](O)C(CO)O2)cc1I</smiles>
$\mathrm{Na}_{2} \mathrm{PdCl}_{4}(0.01-0.5 \mathrm{~mol} \%)$

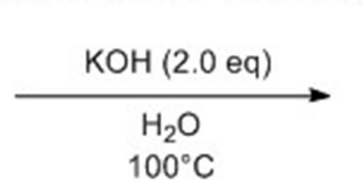

Scheme 1 | Synthesis of 5-phenyl-2' -deoxyuridine from 5-iodo analog (Gallagher-Duval et al., 2013).<smiles>[R]C=C[C@@H]1C[C@H](O)[C@H](CO)O1</smiles>

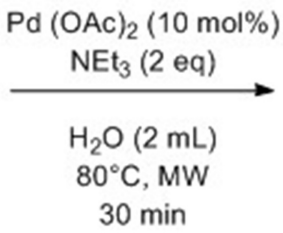<smiles>[R]C=CC1=Cn2c(=O)[nH]c(=O)n2CC1CO</smiles>

Scheme 2 | Synthesis of 5-alkenyl-2'-deoxyuridine from 5-iodo analog (Hervé and Len, 2014).

\section{MICROWAVES ASSISTED HECK CROSS-COUPLINGS IN PURE WATER}

Aqueous Heck cross-couplings applied to classical substrates are well documented (Casalnuovo and Calabrese, 1990; Fihri and Len, 2010; Polshettiwar et al., 2010; Fihri et al., 2011). However, only few recent examples of this reaction performed on nucleosides in aqueous conditions have been reported and none of them used microwaves irradiation assistance.

In 2014, for the first time, Hervé and Len (2014) described a ligand-free Heck cross-coupling reaction under assistedmicrowaves in pure water performed on 5-iodo-2' -deoxyuridine and various acrylate derivatives. Reactions were carried out using $\mathrm{Pd}(\mathrm{OAc})_{2}(10 \mathrm{~mol} \%)$ with $\mathrm{Et}_{3} \mathrm{~N}(2 \mathrm{eq})$ as base at $80^{\circ} \mathrm{C}$ (Scheme 2).

The reported method proceeded well with the acrylate having shortest chains since it permitted to provide the desired compounds in $90 \%$ yields. Reactions starting from more lipophilic esters were not so efficient and led to moderate yields (35$45 \%$ ). The presence of a heteroatom (such as chlorine or oxygen) did not seem to change the hydrophobic/lipophilic balance of the reactant; consequently cross-coupling compounds were isolated in the same moderate range of yields. In order to have a large variety of compounds, substitution of the ester by amido and cyano groups was studied. Application of the methodology allowed access to the target compounds in respectively, 60 and $51 \%$ yields. The authors were able to synthesize the well-known antiviral BVDU in three successive totally aqueous steps: free-ligand microwaves-assisted Heck cross-coupling of 5-iodo-2'-deoxyuridine with methylacrylate; hydrolysis and Hunsdiecker reaction. Finally, this new methodology permitted to isolate BVDU with a better overall yield than that reported in the literature (56 vs. 31\%) (De Clercq et al., 1986).

\section{CONCLUDING REMARKS}

Conventional syntheses of cross-coupling reactions for the preparation of new antiviral and antitumor nucleoside analogs have been well reported. During the last decade, the new synthetic objectives in this area were to design and to improve materials, products processes and systems. In this context, a challenging new avenue has involved the use of green solvents, catalysts, alternative technologies and energy efficiency. Few groups have already started to search in this domain leading to efficient green reactions. To date, among this scientific community, Len's group proposed the greenest process: efficient reactions starting from unprotected nucleosides with small loadings of palladium in the absence of ligand, in water, under microwave irradiations.

\section{REFERENCES}

Agrofoglio, L. A., Gillaizeau, I., and Saito, Y. (2003). Palladium-assisted routes to nucleosides. Chem. Rev. 103, 1875-1916. doi: 10.1021/cr010374q

Casalnuovo, A. L., and Calabrese, J. (1990). Palladium-catalyzed alkylations in aqueous media. J. Am. Chem. Soc. 112, 4324-4330. doi: 10.1021/ja0016 $7 \mathrm{a} 032$

De Clercq, E., Desgrange, C., Herdewijn, P., Sim, I. S., Jones, A. S., McLean, M. J., et al. (1986). Synthesis and antiviral activity of (E)-5(2-bromovinyl)uracil and (E)-5-(2-bromovinyl)uridine. J. Med. Chem. 29, 213-217. doi: 10.1021/jm00152a008

Fihri, A., and Len, C. (2010). "Microwave assisted coupling reactions in aqueous media," in Aqueous Microwave Chemistry - Synthesis and Catalysis, eds V. Polshettiwar and R. S. Varma (Cambridge, UK: RSC Green Chemistry Series, RSC Publishing), 55-90.

Fihri, A., Luart, D., Len, C., Solhi, A., Chevrin, C., and Polshettiwar, V. (2011). Suzuki-Miyaura cross-coupling reactions with low catalyst loading: a green and sustainable protocol in pure water. Dalton Trans. 40, 3116-3121. doi: 10.1039/c0dt01637c

Fresneau, N., Hiebel, M. A., Agrofoglio, L. A., and Berteina-Raboin, S. (2012). Efficient synthesis of unprotected C-5-aryl/heteroaryl-2'-deoxyuridine via a Suzuki-Miyaura reaction in aqueous media. Molecules 17, 14409-14417. doi: 10.3390/molecules 171214409 
Gallagher-Duval, S., Hervé, G., Enderlin, G., and Len, C. (2013). Improved microwave-assisted ligand-free Suzuki-Miyaura cross-coupling of 5iodo-2'-deoxyuridine in pure water. New J. Chem. 37, 1989-1995. doi: 10.1039/c3nj00174a

Hervé, G., and Len, C. (2014). First ligand-free, microwave-assisted, Heck crosscoupling reaction in pure water on a nucleoside - Application to the synthesis of antiviral BVDU. RSC Adv. 4, 46926-46929. doi: 10.1039/C4RA09798J

Polshettiwar, V., Decottignies, A., Len, C., and Fihri, A. (2010). Suzuki-Miyaura cross-coupling reactions in aqueous media: green and sustainable syntheses of biaryls. ChemSusChem 5, 502-522. doi: 10.1002/cssc.200900221

Conflict of Interest Statement: The authors declare that the research was conducted in the absence of any commercial or financial relationships that could be construed as a potential conflict of interest.
Received: 15 December 2014; accepted: 23 January 2015; published online: 17 February 2015.

Citation: Hervé G and Len C (2015) Aqueous microwave-assisted cross-coupling reactions applied to unprotected nucleosides. Front. Chem. 3:10. doi: 10.3389/fchem. 2015.00010

This article was submitted to Medicinal and Pharmaceutical Chemistry, a section of the journal Frontiers in Chemistry.

Copyright (c) 2015 Hervé and Len. This is an open-access article distributed under the terms of the Creative Commons Attribution License (CCBY). The use, distribution or reproduction in other forums is permitted, provided the original author(s) or licensor are credited and that the original publication in this journal is cited, in accordance with accepted academic practice. No use, distribution or reproduction is permitted which does not comply with these terms. 